\title{
Le dimensioni motivazionali dell'apprendimento scolastico: uno studio correlazionale sul concetto di sé e gli stili di attribuzione
}

\author{
Andrea Caputo
}

Sapienza Università di Roma - Department of Dynamic and Clinical Psychology (Italy)

doi: 10.7358/ecps-2015-012-capu

andrea.caputo@uniroma1.it

\section{THE MOTIVATIONAL PATTERNS OF SCHOOL LEARNING: A CORRELATIONAL STUDY ON SELF-CONCEPT AND ATTRIBUTIONS}

\section{Abstract}

The paper proposes a correlational study on 18436 seventh grade students from lower secondary schools of nine Italian regions, which explores some motivational patterns affecting learning. In detail, it has two research aims: (1) to examine differences by gender and regularity of grade attendance in Math and Reading self-concept and in causal attributions to explain success or failure in learning situations; (2) to test the association between Math/Reading self-concept and causal attributions, not depending on gender and regularity of grade attendance. Data were collected by the "Student Background Questionnaire» implemented by the National Evaluation Service. With regard to the first research aim, results highlight that male students are characterized by higher Math self-concept and external, stable and uncontrollable attributions to explain success or failure; instead, female students show higher Reading self-concept and effort as causal attribution to explain their academic success. In addition, repeaters overall show lower academic self-concept and less functional attributions. With regard to the second research aim, results confirm the strong association between self-concept and attributions even controlling for gender and regularity of grade attendance. The present study contributes to the development of research on motivational patterns of learning and to the detection of potential protective or risk factors affecting school success or failure.

Keywords: Academic self-concept, Causal attributions, Correlational study, Regularity of grade attendance, School learning. 


\section{INTRODUZIONE}

Diverse teorie psicologiche evidenziano come il concetto di apprendimento sia strettamente connesso a quello di motivazione in quanto rimanda all'acquisizione di nuove conoscenze e di nuovi sistemi di riferimento per rapportarsi più efficacemente all'ambiente. In tal senso, l'apprendimento può essere concettualizzato quale processo di cambiamento sostenuto dai bisogni e dalle spinte motivazionali che orientano il pensiero, il comportamento e il raggiungimento di mete.

La nota "piramide dei bisogni» di Maslow (1954) evidenzia come la spinta all'apprendimento rientri in una più generale motivazione alla riuscita e all'autorealizzazione dell'individuo che può esprimersi solo a patto che egli senta soddisfatte alcune esigenze motivazionali di base legate al bisogno di sicurezza e protezione. Per Hoppe (1930) la motivazione all'apprendimento è orientata da ciò che l'individuo si propone di fare ovvero dal suo livello di aspirazione che risulta dal compromesso fra due opposte tendenze: il desiderio di evitare una delusione associata all'insuccesso, che riduce il livello di aspirazione, e il desiderio di avere successo che porta invece a porsi mete più elevate. Secondo tale prospettiva, per uno studente la situazione di apprendimento ideale consisterebbe nel mantenere un livello realistico di aspirazioni sufficientemente stimolanti, ma non irraggiungibili per la proprie possibilità. A questo si aggiungono le teorie motivazionali di Atkinson e McClelland (McClelland et al., 1953), le quali evidenziano come la motivazione nei confronti di una prestazione dipenda dalla percezione che la persona ha della difficoltà del compito e dalle risorse che ritiene di poter mettere in campo. Per McClelland gli individui con elevata motivazione alla riuscita (rispecchiante il desiderio di successo e la paura del fallimento) - la quale fa parte dei tre sistemi motivazionali di base dell'agire umano insieme alla motivazione all'affiliazione e al potere - tendono a porsi mete più realistiche nell'apprendimento. Per Atkinson, inoltre, le differenze individuali nella motivazione alla riuscita si spiegano in riferimento ad un bisogno costante di evitare l'insuccesso, che risulta tanto più intenso quanto più le mete sono al di sopra delle proprie possibilità e numerosi sono gli insuccessi passati. Infine, Bonaiuto (1967) propone un modello teorico per lo studio dei nuclei motivazionali ad impronta fenomenologica e dinamica che consente di individuare una serie di mete ben distinguibili nel campo di azione del soggetto. Si delineano nove sistemi principali di motivazioni e rispettivi oggetti-meta, intesi come oggetti o scopi raggiunti i quali vi è la soddisfazione o gratificazione motivazionale (Bonaiuto, 1967; Biasi, 2010). Tra questi, è specificamente annoverata la motivazione alla conoscenza avente come finalità sostanziale la raccolta di informazioni e l'edificazione di concetti o schemi mentali. Essa comprende 
aspetti della conoscenza ordinata, tipicamente riferita alle abilità cognitive convergenti (ossia alla capacità di risolvere problemi che ammettono una sola risposta esatta) e della conoscenza variata, in particolare gli aspetti della esplorazione e della fantasia (ossia alla capacità di fornire molteplici soluzioni a problemi che ammettono più soluzioni).

Seppure con delle differenze, tutte queste teorie evidenziano la stretta interdipendenza tra processi cognitivi ed emotivi e l'importanza di considerare la motivazione e la paura dell'insuccesso nel predisporre esperienze di apprendimento. Ciò al fine di non compromettere la soddisfazione dei bisogni di base e proporre mete realistiche e al contempo sufficientemente stimolanti.

A tale riguardo, le convinzioni che gli studenti hanno rispetto alla propria capacità di controllare gli eventi sembrano giocare un ruolo significativo nell'autoregolazione del proprio processo di apprendimento e nell'esito delle proprie performances scolastiche (Weiner, 1985; Bandura, 1986; Zimmerman, 1989; Schunk, 1991). L'apprendimento e il buon esito di una carriera scolastica sono infatti influenzati, a parità di capacità cognitive, dalla motivazione e dal concetto di sé che l'allievo ha circa le proprie competenze e capacità (Inglehart et al., 1989; Hair \& Graziano, 2003; Meleddu \& Scalas, 2009). In ambito educativo il concetto di sé è stato associato alla motivazione, alle aspirazioni personali a lungo termine e ai risultati scolastici, come riportato da numerosi studi longitudinali (Marsh \& Craven, 2006; Marsh, 2007; Marsh \& O’Mara, 2008; Marsh \& Scalas, 2010). Esso corrisponde a una valutazione complessiva delle proprie capacità, e si distingue, quindi, dall'autoefficacia per la generalità (Covington, 1998). Chi ha un buon concetto di sé è in genere più motivato e possiede approcci più positivi alle diverse situazioni, mentre chi è incerto circa il proprio concetto di sé oppure teme che altri lo vedano diverso da come lui stesso si rappresenta, tende a mettere in atto strategie difensive, quali i self-handicap (Arkin \& Baumgardner, 1985; Midgley et al., 1997). Come sostengono Moè e De Beni (2002), per self-handicap si intendono gli ostacoli reali o fittizi che vengono anticipati all'esecuzione di un compito al fine di massimizzare il concetto di sé in caso di successo o per mantenerlo intatto in situazioni di insuccesso (ad esempio, giustificare un basso voto riportato in una prova affermando l'eccessiva difficoltà del compito). La stretta relazione tra attribuzioni causali, apprendimento strategico e successo, è inoltre dimostrata empiricamente (ClaytonJones et al., 1992; Chan, 1994; Marsh et al., 1999; Marsh \& Hattie, 2006), in quanto la presenza di buone prestazioni e di reazioni positive da parte degli studenti contribuisce a rafforzare la sicurezza in se stessi (Baumeister et al., 2003). A tale riguardo, l'apprendimento strategico è considerato come il prodotto di due dimensioni metacognitive connesse tra loro: le riflessioni 
personali rispetto alle proprie conoscenze e abilità (self appraisal) e le azioni cognitive che guidano il pensiero come la pianificazione, la regolazione, il monitoraggio, la valutazione (self management). Un basso concetto di sé scolastico può, in tal senso, indurre una percezione di scarsa auto-efficacia, cioè l'impressione di non poter modificare il corso degli eventi, dal momento che l'auto-percezione di uno studente è spesso collegata alle proprie strutture di attribuzione (Johnson, 1981). Una bassa autostima e una scarsa motivazione allo studio rivestono, infatti, un ruolo decisivo nei casi di rendimento scadente, reazioni eccessive alle difficoltà e agli insuccessi, abbandono scolastico e disagio educativo più in generale (Marsh et al., 1999).

Le teorie sull'attribuzione causale sostengono che, di fronte a un successo o a un fallimento scolastico, il soggetto può mettere in atto differenti schemi di spiegazione. Al fine di mantenere o incrementare la motivazione allo studio e la spinta a migliorare è necessario, quindi, che un ragazzo possa ricondurre i propri esiti scolastici ad aspetti che ritiene di poter controllare, possibilmente interni, personali, suscettibili di cambiamento. Rappresentano elementi con queste caratteristiche, ad esempio, lo sforzo, l'impegno, il cambiamento di strategie di studio inadeguate, l'impiego di nuovi approcci di lavoro, il superamento di ostacoli quali la presenza di lacune pregresse o il miglioramento di abilità di base quando siano da rafforzare.

In merito al rapporto tra stili di attribuzione e concetto di sé in ambito scolastico, numerosi studi hanno riscontrato che studenti con un elevato concetto di sé tendono ad attribuire i propri successi a fattori interni, come l'abilità e l'impegno, mentre studenti con un basso concetto di sé sono più propensi a riconoscere cause esterne all'origine dei risultati positivi di apprendimento (Ickes \& Layden, 1978; Marsh et al., 1984). Si supporta l'idea che, a fronte di possibili insuccessi, studenti con elevato concetto di sé scolastico ricorrano più frequentemente a cause di attribuzione esterne, mentre un basso concetto di sé porti a internalizzare la responsabilità per i propri fallimenti. Tale fenomeno, definito bias edonico o effetto self-serving (Marsh et al., 1984), per cui si scelgono cause di tipo interno per il successo e cause di tipo esterno per giustificare il fallimento nelle prestazioni scolastiche conseguite, sembra riflettere il tentativo di proteggere l'immagine di sé e potenziare così la propria autostima. Marsh e collaboratori (1984) hanno inoltre rilevato che le attribuzioni di abilità in Matematica risultano fortemente correlate a un maggiore concetto di sé in tale ambito disciplinare rispetto all'Italiano, e viceversa, sottolineando come vi sia una relazione significativa tra i due costrutti in riferimento a specifiche aree e domini di apprendimento.

Da ciò nasce, quindi, la necessità che si sviluppi e si consolidi la cultura di una valutazione autentica, basata sul contributo di autovalutazione degli 
alunni, in modo da favorire una realistica conoscenza di sé e promuovere processi motivazionali capaci di sostenere lo sviluppo delle potenzialità individuali per l'apprendimento.

\section{OBIETTIVI DI RICERCA}

Il presente studio intende esplorare alcune dimensioni motivazionali coinvolte nel processo di apprendimento in studenti di scuola secondaria di primo grado.

Nello specifico, esso si pone due obiettivi di ricerca:

1. Verificare la presenza di eventuali differenze in funzione del genere e della regolarità negli studi relativamente ai seguenti costrutti:

- concetto di sé in Matematica;

- concetto di sé in Italiano;

- cause di attribuzione (fortuna, aiuto, facilità del compito, abilità e impegno) in situazioni di successo e insuccesso scolastico;

- fattori di attribuzione (locus of control, controllabilità, stabilità) in situazioni di successo e insuccesso scolastico.

2. Esaminare la correlazione esistente tra il concetto di sé in Matematica e Italiano e le specifiche modalità di attribuzione in situazioni di successo e insuccesso scolastico, e testare la robustezza di tale associazione al netto delle differenze imputabili al genere e alla regolarità negli studi.

\section{Metodi}

\subsection{Partecipanti}

Il campione utilizzato per il presente studio è costituito da 18436 studenti $($ maschi $=51 \%$; femmine $=49 \%)$ con una età media di 11.93 anni $(D S=$ 0.418). Gli studenti appartengono a 1128 classi seconde di 327 scuole secondarie di primo grado collocate in nove regioni italiane (Calabria, Campania, Emilia Romagna, Lombardia, Marche, Piemonte, Puglia, Sicilia, Veneto). 


\subsection{Dati e strumenti}

I dati sono stati rilevati nell'ambito della valutazione del progetto nazionale Qualità e Merito (PQM) ${ }^{1}$ condotta da INVALSI ${ }^{2}$ nell'anno scolastico 2010/2011. Le misure utilizzate sono tratte dal Questionario Studente predisposto da INVALSI a corredo delle prove di apprendimento per la raccolta delle informazioni di background dello studente circa le attività svolte a scuola e quelle praticate nel tempo libero, le opinioni e gli atteggiamenti in merito alla scuola e allo studio, utili ai fini dell'interpretazione dei risultati scolastici.

\subsection{Misure}

\subsubsection{Il concetto di sé scolastico in Matematica e Italiano}

Il concetto di sé scolastico include tutti i pensieri dello studente su se stesso e sulla conoscenza circa le proprie competenze e capacità ed è definito a partire dalle esperienze acquisite in determinati ambiti di apprendimento e in specifiche materie scolastiche. Tra le cognizioni riferite a se stessi, il concetto di sé è quello che organizza tutto ciò che noi crediamo di essere, cosa pensiamo di essere in grado di fare, e quanto bene pensiamo di saperlo fare. E la parte informativa della concezione di sé, ovvero quello che noi sappiamo o crediamo di noi stessi (Schmeck, 1988), la cui componente affettiva risulta meglio definita dal costrutto dell'autostima.

Nel Questionario Studente il concetto di sé scolastico viene esplorato utilizzando una domanda derivata dall'indagine Trends in International Mathematics and Science Study (TIMSS) del 2007 (Mullis et al., 2008), declinandola sia per la Matematica che per l'Italiano. Il concetto di sé in Italiano e Matematica viene costruito a partire da tre diversi aspetti: la performance (essere bravi), il processo di apprendimento (imparare facilmente), e il confronto con il gruppo di riferimento nel valutare la propria performance (Marsh et al., 1988).

Nel presente studio, ciascuna dimensione è indagata con una batteria di 3 items valutati su una scala likert a 4 passi (da "per niente d'accordo" a «molto d'accordo»), che mostra una valida consistenza interna sia per la

1 Il progetto PQM è finanziato con i fondi dell'Unione Europea - PON Istruzione 2007-2013 (A-2-FSE-2009-2) ed è volto al miglioramento dei processi di insegnamento/apprendimento in Italiano e Matematica nelle scuole secondarie di primo grado.

2 Istituto Nazionale per la Valutazione del Sistema Educativo di Istruzione e di Formazione (INVALSI). Le opinioni espresse in questo articolo sono da attribuirsi all'autore e non impegnano la responsabilità dell'Istituto. 
Matematica $(\alpha=.795)$ che per l'Italiano $(\alpha=.788)$. Inoltre, l'analisi fattoriale delle componenti principali condotta su ciascuna batteria conferma la struttura unidimensionale delle scale, spiegando rispettivamente il $71.33 \%$ (Matematica) e il 70.67\% (Italiano) della varianza complessiva.

\subsubsection{Gli stili di attribuzione}

Per attribuzioni intendiamo le spiegazioni che un soggetto fornisce per i risultati delle sue azioni, in rapporto alle credenze di causalità degli eventi. Vengono generalmente distinte cinque cause di attribuzione in caso di successo e insuccesso nell'apprendimento, a cui corrispondono differenti pattern cognitivi, affettivi e motivazionali: aiuto esterno, abilità personale, facilità del compito, fortuna/caso e impegno personale (Weiner et al., 1971; Weiner et al., 1979). Lo stile di attribuzione prevede, inoltre, tre dimensioni lungo cui le cause del successo o dell'insuccesso possono variare:

- l'attribuzione a fattori interni o esterni all'individuo (locus of control);

- l'attribuzione a fattori stabili o instabili (percezione da parte del soggetto del perdurare delle cause);

- l'attribuzione a fattori controllabili o incontrollabili (percezione della responsabilità personale dei comportamenti prodotti).

A tale riguardo, come osserva Marini (1990), il locus of control è legato soprattutto all'autostima; la stabilità è in rapporto con le aspettative di una persona dopo un successo o un insuccesso; il controllo si riferisce invece all'influenza che il soggetto (o altri) può esercitare sugli eventi. Nel Questionario Studente le attribuzioni sono indagate con una batteria adattata da alcuni strumenti italiani ${ }^{3}$ costituita da dieci items, quattro dei quali riferiti a situazioni di successo (es. "In una discussione in classe il tuo intervento è stato molto apprezzato») e sei a situazioni di insuccesso (es. "Sei alla fine dell'anno scolastico e hai molte insufficienze»), che possono presentarsi nelle attività scolastiche. Ogni item prevede cinque modalità di risposta, ognuna indicante una specifica scelta attributiva. Nel presente studio, la batteria mostra una buona consistenza interna, con un Alpha di Cronbach pari a .843, che risulta mantenuta a livelli accettabili sia per gli item riferiti a situazioni di successo $(\alpha=.675)$ che di insuccesso $(\alpha=.803)$ scolastico. A partire dalla struttura fattoriale della batteria, definita in funzione del quadro teorico del costrutto in esame (Tabella 1), sono state costruite 16 variabili che, per ciascuno studente, permettono di rilevare il differente ricorso alle modalità di

3 La domanda inserita nel questionario per la scuola secondaria di I grado è tratta da Cornoldi et al., 1996. 
attribuzione proposte (aiuto esterno, facilità/difficoltà del compito, fortuna/ caso, abilità personale, impegno personale) e i principali fattori di attribuzione (internalità, stabilità e controllabilità) rispettivamente in situazioni di successo e insuccesso scolastico.

Tabella 1. - Possibili cause della prestazione in un compito.

\begin{tabular}{cccc}
\hline & & Controllabile & INCONTROLLABILE \\
\hline \multirow{2}{*}{ INTERNA } & Stabile & & Abilità personale \\
& Instabile & Impegno personale & \\
Esterna & Stabile & & Facilità del compito \\
& Instabile & Aiuto esterno & Fortuna/caso \\
\hline
\end{tabular}

\subsection{Procedure di analisi}

Le procedure di analisi sono state condotte attraverso il software statistico SPSS 17.0.

Il Test T di Student per campioni indipendenti è stato utilizzato al fine di valutare la presenza di eventuali differenze di genere (femmine versus maschi) nei costrutti in esame, mentre il test della varianza (ANOVA ad una via) ha permesso di verificare l'ipotesi di indipendenza in funzione della regolarità negli studi. A tale riguardo, sono state individuate tre specifiche categorie: la prima contempla gli alunni «anticipatari» di almeno un anno rispetto al proprio percorso scolastico (10-11 anni), la seconda gli alunni «regolari» rispetto all'età normalmente prevista per la frequenza della classe seconda nella scuola secondaria di primo grado (12 anni) e, infine, $\mathrm{i}$ "posticipatari», ovvero gli alunni in ritardo di almeno un anno rispetto al normale percorso scolastico (dai 13 anni in su). Per valutare le differenze nei punteggi ottenuti tra i diversi sottogruppi di studenti, l'ipotesi di uguaglianza delle varianze è stata verificata con il test di Levène. Inoltre, sono stati presi in considerazione i punteggi standardizzati in modo da garantire una migliore confrontabilità delle misure utilizzate.

Infine, è stata effettuata una analisi della correlazione parziale (coefficiente $R$ di Pearson) tra le misure di attribuzione e il concetto di sé scolastico in Matematica e Italiano. In questo caso il test è stato definito a una coda, dal momento che si ipotizza che un migliore concetto di sé scolastico sia associato a modalità di attribuzione più funzionali, caratterizzate da una più elevata internalità e controllabilà delle cause di successo e insuccesso scolastico. Al fine di testare la robustezza dei risultati, sono stati utilizzati alcuni modelli di regressione lineare per valutare la capacità predittiva degli stili di attribuzione (variabili dipendenti) sulla base del concetto di sé in Italiano e Matematica 
(variabili esplicative), controllando i punteggi per le differenze imputabili al genere e alla regolarità negli studi.

\section{Risultati}

Di seguito sono riportate le caratteristiche descrittive del campione in merito al genere, alla regolarità negli studi e alla distribuzione per regione (Tabella 2).

Tabella 2. - Caratteristiche del campione di studenti per genere, regolarità negli studi e regione di appartenenza.

\begin{tabular}{|c|c|c|c|c|}
\hline Genere & FREQUENZA & Percentuale & $\begin{array}{l}\text { Percentuale } \\
\text { VAlida }\end{array}$ & $\begin{array}{c}\text { Percentuale } \\
\text { CUMulata }\end{array}$ \\
\hline Femmina & 9031 & 49 & 49 & 49 \\
\hline Maschio & 9403 & 51 & 51 & 100 \\
\hline Dato mancante & 2 & 0 & 0 & 100 \\
\hline Totale & 18436 & 100 & 100 & \\
\hline $\begin{array}{l}\text { REGOLARITÀ } \\
\text { NEGLI STUDI }\end{array}$ & FREQUENZA & Percentuale & $\begin{array}{c}\text { Percentuale } \\
\text { VAlida }\end{array}$ & $\begin{array}{c}\text { Percentuale } \\
\text { CUMUlata }\end{array}$ \\
\hline Regolare & 17189 & 93.2 & 93.2 & 93.2 \\
\hline Anticipatario & 373 & 2 & 2 & 95.3 \\
\hline Posticipatario & 853 & 4.6 & 4.6 & 99.9 \\
\hline Dato mancante & 21 & 0.1 & 0.1 & 100 \\
\hline Totale & 18436 & 100 & 100 & \\
\hline Regione & FREQUENZA & Percentuale & $\begin{array}{l}\text { Percentuale } \\
\text { Valida }\end{array}$ & $\begin{array}{c}\text { Percentuale } \\
\text { CUMUlata }\end{array}$ \\
\hline Calabria & 1492 & 8.1 & 8.1 & 8.1 \\
\hline Campania & 4768 & 25.9 & 25.9 & 34 \\
\hline Emilia Romagna & 1192 & 6.5 & 6.5 & 40.4 \\
\hline Lombardia & 718 & 3.9 & 3.9 & 44.3 \\
\hline Marche & 544 & 3 & 3 & 47.3 \\
\hline Piemonte & 1117 & 6.1 & 6.1 & 53.3 \\
\hline Puglia & 4657 & 25.3 & 25.3 & 78.6 \\
\hline Sicilia & 3503 & 19 & 19 & 97.6 \\
\hline Veneto & 445 & 2.4 & 2.4 & 100 \\
\hline Totale & 18436 & 100 & 100 & \\
\hline
\end{tabular}




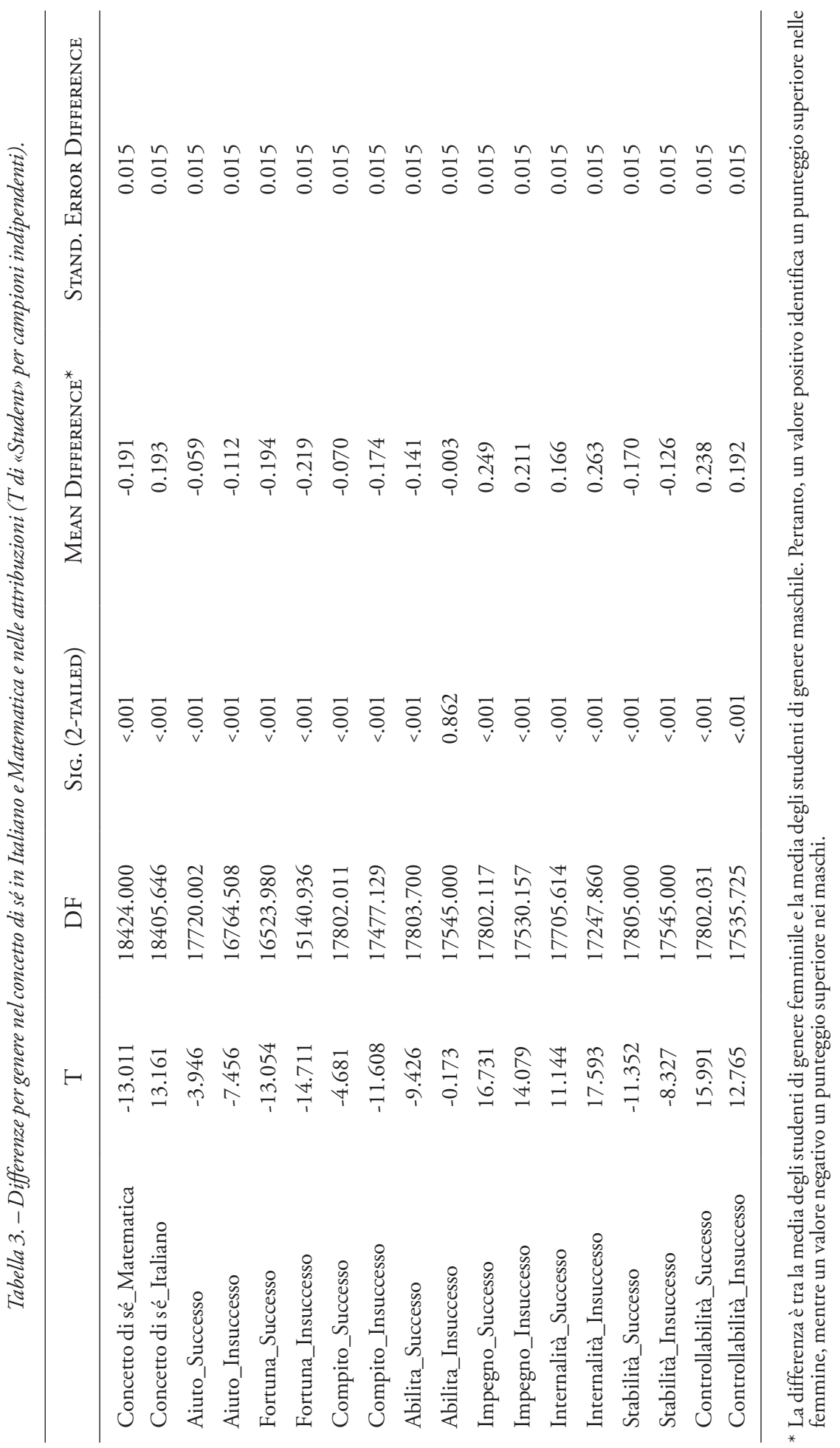


In Tabella 3 sono riportati i risultati del test T di Student per campioni indipendenti per valutare le differenze tra femmine e maschi nei punteggi relativi al concetto di sé in Matematica e Italiano e agli stili di attribuzione. In particolare, si rilevano le seguenti differenze statisticamente significative $(p<0.05)$ : Concetto di sé scolastico

- I maschi presentano un migliore concetto di sé in Matematica.

- Le femmine presentano un migliore concetto di sé in Italiano.

Cause di attribuzione

- I maschi ricorrono più frequentemente all'aiuto esterno, alla fortuna/caso e alla facilità/difficoltà del compito nello spiegare sia i propri successi che i propri insuccessi scolastici, mentre l'abilità viene maggiormente individuata come possibile causa solo in caso di riuscita.

- Le femmine individuano più frequentemente l'impegno come possibile causa delle loro prestazioni scolastiche, sia in situazioni di successo che di insuccesso.

Fattori di attribuzione

- I maschi evidenziano una minore internalità e controllabilità e una maggiore stabilità delle cause in situazioni di successo e insuccesso scolastico rispetto alle femmine.

In Tabella 4 sono ripotati i risulati del test ANOVA per la rilevazione delle differenze nelle dimensioni esaminate in funzione della regolarità negli studi. I test post-hoc hanno inoltre permesso di valutare la direzione delle eventuali differenze emerse come statisticamente significative. In dettaglio, si evidenzia che: Concetto di sé scolastico

- Gli studenti posticipatari presentano un più basso concetto di sé in Matematica e Italiano rispetto agli studenti anticipatari e regolari.

Cause di attribuzione

- Gli studenti posticipatari presentano un maggiore ricorso all'aiuto esterno, alla fortuna/caso e alla facilità/difficoltà del compito e un minore ricorso all'impegno per spiegare i propri successi o insuccessi scolastici rispetto agli studenti anticipatari e regolari.

- Gli studenti posticipatari attribuiscono alla mancanza di abilità l'origine dei propri insuccessi in misura maggiore rispetto agli studenti regolari.

- Gli studenti anticipatari attribuiscono i successi scolastici all'impegno e alla facilità del compito in misura maggiore degli studenti regolari (e posticipatari).

Fattori di attribuzione

- Gli studenti posticipatari riconoscono cause esterne, instabili e incontrollabili all'origine dei propri successi o insuccessi scolastici in misura maggiore degli studenti anticipatari e regolari.

- Gli studenti anticipatari presentano un maggiore locus of control interno nella spiegazione dei propri successi o insuccessi scolastici rispetto agli studenti regolari (e posticipatari). 
Tabella 4. - Differenze per regolarità negli studi (anticipatari, regolari, posticipatari) nel concetto di sé in Italiano e Matematica e nelle attribuzioni (ANOVA).

\begin{tabular}{|c|c|c|c|c|c|c|}
\hline & & Sum of SQuares & df & MEAN SQUare & $\mathrm{F}$ & SIG. \\
\hline \multirow{3}{*}{ Concetto di sé_Matematica } & Between Groups & 102.342 & 2 & 51.171 & 51.610 & $<.001$ \\
\hline & Within Groups & 18247.353 & 18404 & .991 & & \\
\hline & Total & 18349.695 & 18406 & & & \\
\hline \multirow{3}{*}{ Concetto di sé_Italiano } & Between Groups & 96.440 & 2 & 48.220 & 48.647 & $<.001$ \\
\hline & Within Groups & 18242.319 & 18404 & .991 & & \\
\hline & Total & 18338.759 & 18406 & & & \\
\hline \multirow{3}{*}{ Aiuto_Successo } & Between Groups & 111.426 & 2 & 55.713 & 56.138 & $<.001$ \\
\hline & Within Groups & 17652.410 & 17787 & .992 & & \\
\hline & Total & 17763.836 & 17789 & & & \\
\hline \multirow{3}{*}{ Aiuto_Insuccesso } & Between Groups & 57.414 & 2 & 28.707 & 28.843 & $<.001$ \\
\hline & Within Groups & 17444.356 & 17527 & .995 & & \\
\hline & Total & 17501.770 & 17529 & & & \\
\hline \multirow{3}{*}{ Fortuna_Successo } & Between Groups & 117.783 & 2 & 58.891 & 59.308 & $<.001$ \\
\hline & Within Groups & 17662.141 & 17787 & .993 & & \\
\hline & Total & 17779.924 & 17789 & & & \\
\hline \multirow{3}{*}{ Fortuna_Insuccesso } & Between Groups & 37.950 & 2 & 18.975 & 19.028 & $<.001$ \\
\hline & Within Groups & 17478.068 & 17527 & .997 & & \\
\hline & Total & 17516.018 & 17529 & & & \\
\hline \multirow{3}{*}{ Compito_Successo } & Between Groups & 49.808 & 2 & 24.904 & 24.964 & $<.001$ \\
\hline & Within Groups & 17743.827 & 17787 & .998 & & \\
\hline & Total & 17793.634 & 17789 & & & \\
\hline \multirow{3}{*}{ Compito_Insuccesso } & Between Groups & 46.911 & 2 & 23.456 & 23.504 & $<.001$ \\
\hline & Within Groups & 17490.799 & 17527 & .998 & & \\
\hline & Total & 17537.710 & 17529 & & & \\
\hline \multirow{3}{*}{ Abilita_Successo } & Between Groups & 3.434 & 2 & 1.717 & 1.717 & .180 \\
\hline & Within Groups & 17787.376 & 17787 & 1.000 & & \\
\hline & Total & 17790.810 & 17789 & & & \\
\hline \multirow{3}{*}{ Abilita_Insuccesso } & Between Groups & 6.457 & 2 & 3.229 & 3.228 & $<.05$ \\
\hline & Within Groups & 17527.810 & 17527 & 1.000 & & \\
\hline & Total & 17534.267 & 17529 & & & \\
\hline \multirow{3}{*}{ Impegno_Successo } & Between Groups & 97.666 & 2 & 48.833 & 49.097 & $<.001$ \\
\hline & Within Groups & 17691.450 & 17787 & .995 & & \\
\hline & Total & 17789.116 & 17789 & & & \\
\hline \multirow{3}{*}{ Impegno_Insuccesso } & Between Groups & 93.944 & 2 & 46.972 & $47 \cdot 212$ & $<.001$ \\
\hline & Within Groups & 17437.903 & 17527 & .995 & & \\
\hline & Total & 17531.847 & 17529 & & & \\
\hline \multirow{3}{*}{ Internalità_Successo } & Between Groups & 193.442 & 2 & 96.721 & 97.751 & $<.001$ \\
\hline & Within Groups & 17599.650 & 17787 & .989 & & \\
\hline & Total & 17793.092 & 17789 & & & \\
\hline \multirow{3}{*}{ Internalità_Insuccesso } & Between Groups & 105.544 & 2 & 52.772 & 53.072 & $<.001$ \\
\hline & Within Groups & 17427.785 & 17527 & .994 & & \\
\hline & Total & 17533.329 & 17529 & & & \\
\hline \multirow{3}{*}{ Stabilità_Successo } & Between Groups & $10 \cdot 515$ & 2 & 5.257 & $5 \cdot 261$ & $<.01$ \\
\hline & Within Groups & 17774.806 & 17787 & .999 & & \\
\hline & Total & 17785.321 & 17789 & & & \\
\hline \multirow{3}{*}{ Stabilità_Insuccesso } & Between Groups & 43.158 & 2 & 21.579 & 21.626 & $<.001$ \\
\hline & Within Groups & 17489.120 & 17527 & .998 & & \\
\hline & Total & 17532.278 & 17529 & & & \\
\hline \multirow{3}{*}{ Controllabilità_Successo } & Between Groups & $50 \cdot 965$ & 2 & 25.483 & 25.558 & $<.001$ \\
\hline & Within Groups & 17734.689 & 17787 & .997 & & \\
\hline & Total & 17785.655 & 17789 & & & \\
\hline \multirow{3}{*}{ Controllabilità_Insuccesso } & Between Groups & 66.913 & 2 & 33.457 & 33.575 & $<.001$ \\
\hline & Within Groups & 17465.236 & 17527 & .996 & & \\
\hline & Total & 17532.149 & 17529 & & & \\
\hline
\end{tabular}


Le analisi di correlazione tra il concetto di sé scolastico in Matematica e Italiano e le attribuzioni evidenziano le seguenti relazioni statisticamente sigificative, come riportato in Tabella 5.

Tabella 5. - Correlazioni tra concetto di sé in MatematicalItaliano e attribuzioni.

\begin{tabular}{lcccccc}
\hline & \multicolumn{7}{c}{ ConCETTO DI SÉ IN MATEMATICA } & \multicolumn{2}{c}{ ConcetTo DI SÉ IN ItALIANO } \\
\hline & $\begin{array}{c}\text { Pearson } \\
\text { Correlation }\end{array}$ & $\begin{array}{c}\text { Sig. } \\
\text { (1-tailed) }\end{array}$ & $N$ & $\begin{array}{c}\text { Pearson } \\
\text { Correlation }\end{array}$ & $\begin{array}{c}\text { Sig. } \\
\text { (1-tailed) }\end{array}$ & $N$ \\
\hline Aiuto_Successo & -.123 & $<.001$ & 17809 & -.108 & $<.001$ & 17809 \\
Aiuto_Insuccesso & -.075 & $<.001$ & 17549 & -.086 & $<.001$ & 17549 \\
Fortuna_Successo & -.142 & $<.001$ & 17809 & -.139 & $<.001$ & 17809 \\
Fortuna_Insuccesso & -.045 & $<.001$ & 17549 & -.066 & $<.001$ & 17549 \\
Compito_Successo & -.127 & $<.001$ & 17809 & -.127 & $<.001$ & 17809 \\
Compito_Insuccesso & -.064 & $<.001$ & 17549 & -.097 & $<.001$ & 17549 \\
Abilita_Successo & .096 & $<.001$ & 17809 & .060 & $<.001$ & 17809 \\
Abilita_Insuccesso & -.109 & $<.001$ & 17549 & -.058 & $<.001$ & 17549 \\
Impegno_Successo & .092 & $<.001$ & 17809 & .117 & $<.001$ & 17809 \\
Impegno_Insuccesso & .145 & $<.001$ & 17549 & .142 & $<.001$ & 17549 \\
Internalità_Successo & .208 & $<.001$ & 17809 & .201 & $<.001$ & 17809 \\
Internalità_Insuccesso & .092 & $<.001$ & 17549 & .130 & $<.001$ & 17549 \\
Stabilità_Successo & -.003 & .327 & 17809 & -.035 & $<.001$ & 17809 \\
Stabilità_Insuccesso & -.125 & $<.001$ & 17549 & -.111 & $<.001$ & 17549 \\
Controllabilità_Successo & .059 & $<.001$ & 17809 & .088 & $<.001$ & 17809 \\
Controllabilità_Insuccesso & .132 & $<.001$ & 17549 & .126 & $<.001$ & 17549 \\
\hline
\end{tabular}

Rispetto alle cause di attribuzione, gli studenti con un più elevato concetto di sé in Matematica e Italiano:

- Attribuiscono all'abilità e all'impegno le principale cause dei propri successi e insuccessi scolastici.

- Attribuiscono in misura minore i propri successi o insuccessi scolastici all'aiuto esterno, alla fortuna/caso e alla facilità/difficoltà del compito.

In merito ai fattori di attribuzione, un più alto concetto di sé in Matematica e Italiano è associato a una maggiore internalità e controllabilità delle cause dei propri successi e insuccessi scolastici. Rispetto alla stabilità delle cause, invece, un più alto concetto di sé in Italiano è associato a cause instabili riconosciute all'origine dei propri successi o insuccessi. Per il concetto di sé in Matematica ciò vale solo per le situazioni di insuccesso. 
Le analisi di regressione lineare hanno permesso di verificare la tenuta di tale relazione, al netto delle differenze imputabili al genere e alla regolarità negli studi, assumendo il concetto di sé in Italiano e Matematica come variabili indipendenti e le cause e i fattori di attribuzione come variabili dipendenti. I risultati (Tabella 6) confermano a pieno quanto già emerso dalle analisi di correlazione. Complessivamente, i coefficienti di regressione standardizzati $(\beta)$ sono leggermente più elevati per il concetto di sé in Matematica, a segnalare una più forte associazione tra tale costrutto e le modalità di attribuzione in situazioni di apprendimento. In dettaglio, confrontando tra loro i coefficienti, un più alto concetto di sé - sia in Matematica che in Italiano - sembra predire in misura maggiore il ricorso all'impegno per la spiegazione dei propri successi e insuccessi e la tendenza a riconoscere cause interne all'origine dei propri successi.

Tabella 6. - Risultati delle analisi di regressione sulle attribuzioni (variabili dipendenti) in funzione del concetto di sé in Matematica e Italiano (variabili esplicative).

\begin{tabular}{lcccc}
\hline & \multicolumn{2}{c}{$\begin{array}{c}\text { CONCETTO DI SÉ } \\
\text { IN MATEMATICA }\end{array}$} & \multicolumn{2}{c}{$\begin{array}{c}\text { CONCETTO DI SÉ } \\
\text { IN ITALIANO }\end{array}$} \\
\hline Aiuto_Successo & $\beta$ & Sig. & $\beta$ & Sig. \\
\hline Aiuto_Insuccesso & -.112 & $<.001$ & -.086 & $<.001$ \\
Fortuna_Successo & -.071 & $<.001$ & -.067 & $<.001$ \\
Fortuna_Insuccesso & -.137 & $<.001$ & -.107 & $<.001$ \\
Compito_Successo & -.051 & $<.001$ & -.044 & $<.001$ \\
Compito_Insuccesso & -.117 & $<.001$ & -.108 & $<.001$ \\
Abilita_Successo & -.063 & $<.001$ & -.076 & $<.001$ \\
Abilita_Insuccesso & .081 & $<.001$ & .059 & $<.001$ \\
Impegno_Successo & -.105 & $<.001$ & -.046 & $<.001$ \\
Impegno_Insuccesso & .093 & $<.001$ & .086 & $<.001$ \\
Internalità_Successo & .142 & $<.001$ & .109 & $<.001$ \\
Internalità_Insuccesso & .194 & $<.001$ & .164 & $<.001$ \\
Stabilità_Successo & .092 & $<.001$ & .099 & $<.001$ \\
Stabilità_Insuccesso & -.009 & .217 & -.022 & $<.01$ \\
Controllabilità_Successo & -.121 & $<.001$ & -.088 & $<.001$ \\
Controllabilità_Insuccesso & .063 & $<.001$ & .063 & $<.001$ \\
\hline
\end{tabular}

Nota: I coefficienti di regressione sono standardizzati e si intendono al netto del genere e della regolarità negli studi. 


\section{Discussione}

I risultati della presente ricerca hanno evidenziato l'esistenza di alcune rilevanti differenze negli stili di attribuzione adottati dagli studenti rispetto alla spiegazione delle proprie prestazioni scolastiche. In particolare, i maschi tendono a riconoscere cause esterne, stabili e incontrollabili alla base dei propri successi e insuccessi, diversamente dai soggetti di genere femminile che valorizzano il ruolo dell'impegno personale per la buona riuscita delle attività di apprendimento. I maschi, inoltre, attribuiscono maggiormente all'abilità la propria riuscita, assumendo uno stile di tipo «negatore» che sembra volto per lo più a deresponsabilizzarsi rispetto all'eventualità di un fallimento (Moè \& De Beni, 2002). Come suggerito dal nome, questo stile si caratterizza per l'espressione di una negazione dell'insuccesso che è vissuto come qualcosa di non dipendente da sé e per evitare il quale è negata ogni possibilità personale, sia essa più legata all'impegno o a competenze e abilità personali (Kernis et al., 1998). Tale modalità risulta disfunzionale all'apprendimento, in quanto lo studente riconosce poca importanza all'impegno e, a fronte di possibili insuccessi, non cerca strategie più adeguate. Al contempo, il successo contribuisce a rafforzare un positivo concetto di sé, se lo studente si sente veramente capace, oppure può sostenere l'idea che le cose possono riuscire anche senza impegnarsi (Smith et al., 2002). Infatti, la tendenza ad attribuire all'abilità solo i propri successi e a non riconoscerla come causa dei propri fallimenti (selfserving bias), sembra suggerire il possibile ruolo protettivo che il concetto di sé esercita per il mantenimento dell'autostima (Forsterling \& Binser, 2002). Come confermato in letteratura (Crandall, 1969; Maccoby \& Jacklin, 1974; Deaux, 1976; Parsons \& Ruble, 1977; Stipek \& Gralinski, 1991; Burgner \& Hewstone, 1993), lo stile negatore sembra essere più frequente nei maschi, piuttosto che nelle femmine, in particolare per compiti e discipline considerate tendenzialmente maschili quali la Matematica (Fennema \& Sherman, 1978; Wolleat et al., 1980; Heller \& Parsons, 1981). Viceversa, le femmine adottano uno stile di tipo strategico, caratterizzato dallo sforzo intenzionale a portare a termine il compito con successo (Atkinson, 1974), in coerenza con quanto riportato in ulteriori studi (Powers \& Wagner, 1984; Lightbody et al., 1996; Georgiou, 1999). Vi è, infatti, un maggiore impegno a migliorare le proprie competenze piuttosto che a dimostrare la propria abilità e, in tal senso, si può ipotizzare una più forte persistenza di fronte alle difficoltà e agli insuccessi, un coinvolgimento più attivo e una maggiore fiducia nelle proprie possibilità. A tale rifuardo, Moè e De Beni (2002) affermano che alla base di questo stile vi è, in genere, la convinzione di essere capace e la conoscenza dei mezzi e delle strategie per riuscire nelle prestazioni future. Allo stesso tempo, però, le femmine attribuiscono più frequentemente i propri fallimenti 
a cause interne e questo potrebbe comportare, in assenza di una sufficiente autostima, una maggiore tendenza a sviluppare nel tempo un ridotto senso di efficacia a fronte di situazioni di insuccesso scolastico (Dweck \& Bush, 1976; Fontayne et al., 2003).

La relazione tra gli stili di attribuzione e la regolarità negli studi ha fornito, inoltre, alcuni spunti interessanti. Complessivamente, gli studenti posticipatari, ovvero più grandi rispetto all'età normalmente prevista rispetto al livello di studi frequentato, privilegiano cause esterne e stabili nella spiegazione dei propri successi e insuccessi. Inoltre, presentano una minore percezione di controllo e un minore impegno nel fronteggiare i propri fallimenti scolastici, in quanto sembrano prefigurare una sostanziale immodificabilità degli eventi. Tale modalità si traduce in un atteggiamento prevalentemente passivo e fatalista, caratterizzato da scarso interesse per l'apprendimento. $\grave{E}$ dimostrato, infatti, come strategie disfunzionali di questo tipo siano associate a ridotte performances scolastiche e predicano una maggiore probabilità di bocciatura e abbandono (Connell et al., 1994; Skinner et al., 1998), eventi che a loro volta possono esercitare un'influenza negativa sulle convinzioni di controllo rispetto alle proprie prestazioni (Boruchovitch, 2004). Inoltre, si evidenzia che gli studenti posticipatari attribuiscono alla mancanza di abilità l'origine dei propri insuccessi in misura maggiore rispetto agli studenti regolari. Ciò potrebbe essere legato alle precedenti esperienze di fallimento (essendo studenti in ritardo scolastico) che hanno contribuito a ridurne l'autostima e incrementarne il sentimento di impotenza appresa (Tse, 2000). Per impotenza appresa intendiamo quella situazione che si instaura quando, a seguito di ripetuti fallimenti attribuiti alla mancanza stabile di capacità o a cause che sono al di fuori del proprio controllo personale, l'individuo impara a essere impotente, passivo e rassegnato di fronte a situazioni simili a quella in cui aveva in precedenza fallito (Abramson et al., 1978). In tal senso, si delinea un circolo vizioso di profezie che si autoavverano per cui la convinzione di non essere capace porta a non impegnarsi e a non studiare ottenendo risultati mediocri che confermano l'originale convinzione dello studente.

$\mathrm{Al}$ contrario, gli studenti anticipatari presentano in misura maggiore un locus of control interno per la spiegazione dei propri successi o insuccessi scolastici e vedono nell'impegno e nella facilità del compito le principali cause di riuscita rispetto ai propri compagni regolari o posticipatari. Probabilmente, il combinato tra impegno e facilità del compito quali principali cause di successo ci lascia supporre che tali studenti siano caratterizzati da una spiccata tendenza al successo (Atkinson, 1965) che li porta a scegliere obiettivi sfidanti, leggermente più difficili rispetto a quelli affrontati in precedenza. L'atteggiamento verso il compito è positivo e si caratterizza per la focalizzazione 
dell'attenzione, la ricerca di adeguate strategie di soluzione e la persistenza di fronte alle difficoltà. Una volta raggiunto il successo la tendenza è quella di attribuire la riuscita al proprio impegno e di valutare il compito come facile.

Un ulteriore dato, confermato dalla letteratura internazionale (Fredericks \& Eccles, 2002; Heyman \& Legare, 2004; Herbert \& Stipek, 2005), concerne la differenza riscontrata nel concetto di sé scolastico, per cui i maschi si riconoscono maggiori abilità in Matematica rispetto alle femmine che, al contrario, si percepiscono più competenti in Italiano. La minore tendenza delle ragazze a identificarsi nel dominio matematico potrebbe essere determinata dalla diffusione di alcuni stereotipi di genere secondo cui la Matematica sarebbe un ambito di applicazione più indicato per i ragazzi (National Science Foundation, 2003; Steele, 2003; Dweck, 2007; Eccles, 2007; Guiso et al., 2008). Di contro, i ragazzi sono più riluttanti a dedicarsi alla lettura al di fuori del consueto orario scolastico come evidenziato dai dati PIRLS (Progress in International Reading Literacy Study; Twist et al., 2007), vedono la lettura come una attività fondamentalmente noisa (Nieuwenhuizen, 2001) e, a differenza delle femmine, attribuiscono ad essa minore importanza quale interesse personale che può essere condiviso con altri e può favorire la socializzazione con i pari (DCSF, 2007). Queste differenze di genere sembrano, quindi, risentire di un bias culturale che porta gli studenti a sviluppare un minore interesse per discipline di studio e future professioni nei domini che ritengono poco compatibili con il concetto di sé (Liben et al., 2001; Malcom et al., 2005; Frome et al., 2006; Killen et al., 2006; Denissen et al., 2007; Newcombe, 2007). Inoltre, anche la regolarità negli studi sembra associata al concetto di sé, con specifico riguardo per gli studenti posticipatari che forniscono una peggiore autovalutazione delle proprie abilità in riferimento a entrambe le materie scolastiche. Numerosi studi hanno dimostrato, infatti, l'influenza negativa che precedenti esperienze di fallimento scolastico (quali la bocciatura) possono esercitare sul concetto di sé, l'autostima e le capacità di coping, con conseguenti problemi di adattamento a lungo termine (Setencich, 1994; Alexander et al., 2003).

I risultati ribadiscono, infine, la forte associazione tra concetto di sé e stili di attribuzione come già evidenziato da studi precedenti (Ickes \& Layden, 1978; Marsh et al., 1984). Gli studenti con più elevato concetto di sé in Matematica e Italiano percepiscono, a tale riguardo, una più efficace capacità di determinare gli esiti del proprio apprendimento (ricorso all'impegno), che sembra veicolare un più forte coinvolgimento e una maggiore autostima alla base del proprio successo scolastico (Arkin et al., 1980). Ciò contribuisce ad adottare strategie più funzionali che a loro volta permettono di agire come rinforzo per le successive attività di apprendimento. Al contrario un più basso concetto di sé è associato a modalità di attribuzione più disfunzionali, 
quali l'aiuto, la fortuna e la difficoltà del compito, così come è riportato in letteratura anche in riferimento a ulteriori costrutti quali la depressione e la scarsa autostima (Haugen \& Lund, 2002; Hirschy \& Morris, 2002).

\section{CONCLUSIONI}

Il presente studio intende contribuire allo sviluppo della ricerca sulle dimensioni motivazionali dell'apprendimento al fine di identificare possibili fattori protettivi o di rischio, rispettivamente per situazioni di successo e insuccesso scolastico. L'evidenza riscontrata suggerisce come le convinzioni che gli studenti hanno rispetto alle proprie abilità e competenze siano fortemente associate alle modalità con cui spiegano i propri esiti scolastici. L'intervento sugli alunni con difficoltà di apprendimento può, in tal senso, partire direttamente dalla percezione che gli studenti hanno di sé in rapporto ai compiti e alle sfide scolastiche con cui si confrontano quotidianamente e alle spiegazioni che adottano per giustificare i propri insuccessi (Banks \& Woolfson, 2008). Numerosi programmi sperimentali hanno già dimostrato la loro efficacia a riguardo per la prevenzione di situazioni a rischio di abbandono o scarso rendimento (Van Overwalle \& De Metsenaere, 1990; Okolo, 1992; Nelson \& Manset-Williamson, 2006). Il ruolo dell'insegnante diventa quindi fondamentale in quanto la sua capacità di rinforzare e valorizzare il concetto di sé dei propri studenti, proporre una visione dell'errore come parte integrante dell'apprendimento e non come fallimento irreversibile, coinvolgere maggiormente gli studenti quali attori principali della scena educativa, può migliorarne la motivazione ad apprendere e incidere direttamente sui loro successivi esiti formativi.

\section{RIFERIMENTI BIBLIOGRAFICI}

Abramson, L. Y., Seligman, M. E. P., \& Teasdale, I. (1978). Learned helplessness in humans: Critique and reformulation. Journal of Abnormal Psychology, 87, 49-59.

Alexander, K. L., Entwisle, D. R., \& Dauber, S. L. (2003). On the success of failure: $A$ reassessment of the effects of retention in the primary grades (2nd ed.). Cambridge: Cambridge University Press.

Arkin, R. M., \& Baumgardner, A. H. (1985). Self-handicapping. In J. H. Harvey \& G. W. Weary (Eds.), Attribution: Basic issues and applications (pp. 169-202). San Diego, CA: Academic Press. 
Arkin, R. M., Appleman, A. J., \& Burger, J. M. (1980). Social anxiety, self-presentation, and the self-serving bias in causal attribution. Journal of Personality and Social Psychology, 38, 23-35.

Atkinson, J. W. (1974). Motivation and achievement. Washington, DC: V. H. Winston and Sons.

Atkinson, J. W. (1965). An introduction to motivation. New York: American BookVan Nostrand-Reinhold.

Bandura, A. (1986). Social foundations of thought and action: A social cognitive theory. Englewood Cliffs, NJ: Prentice Hall.

Banks, M., \& Woolfson, L. (2008). Why do students think they fail? The relationship between attributions and academic self-perceptions. British Journal of Special Education, 35(1), 49-56.

Baumeister, R. F., Campbell, J. D., Krueger, J. I., \& Vohs, K. D. (2003). Does high self-esteem cause better performance, interpersonal success, happiness, or healthier lifestyles? Psychological Science in the Public Interest, 4, 1-44.

Biasi, V. (2010). Processi affettivi e dinamiche della conoscenza. Milano: Guerini.

Bonaiuto, P. (1967). La fenomenologia delle motivazioni nel «design» per l'età evolutiva. Rassegna di Psicologia Generale e Clinica, 8, 36-65.

Boruchovitch, E. (2004). A study of causal attributions for success and failure in mathematics among Brazilian students. Interamerican Journal of Psychology, 38(1), 53-60.

Burgner, D., \& Hewstone, M. (1993). Young children's causal attributions for success and failure: "Self-enhancing» boys and «self-derogating» girls. British Journal of Developmental Psychology, 11, 125-129.

Chan, L. K. S. (1994). Relationship of motivation, strategic learning, and reading achievement in grades 5, 7 and 9. Journal of Experimental Education, 62(3), 235-245.

Clayton-Jones, L., Rodwell, K., Skehan, J., Archer, J., Chan, L., \& Moore, P. (1992). Approaches to learning, attributions, goal orientations and achievement: A developmental perspective. Paper presented at the AARE/NZARE Joint Conference, Geelong, VI.

Connell, J. P., Spencer, M. B., \& Aber, J. L. (1994). Educational risk and resilience in African American Youth: Context, self, and action outcomes in school. Child Development, 65, 493-506.

Cornoldi, C., Gardinale, M., Masi, A., \& Pettenò, L. (1996). Impulsività e autocontrollo. Trento: Erickson.

Covington, M. V. (1998). The will to learn. Cambridge: Cambridge University Press.

Crandall, V. C. (1969). Sex differences in expectancy of intellectual and academic reinforcement. In C. P. Smith (Ed.), Achievement-related motives in children (pp. 11-45). New York: Russell Sage Foundation. 
DCSF (2007). Gender and education: The evidence on pupils in England. London: DCSF.

Deaux, K. (1976). Sex: A perspective on the attribution process. In J. H. Harvey, W. J. Ickes, \& R. F. Kidd (Eds.), New directions in attribution research, Vol. 1 (pp. 335-352). Hillsdale, NJ: Erlbaum.

Denissen, J. J. A., Zarrett, N. R., \& Eccles, J. S. (2007). I like to do it, I'm able, and I know I am: Longitudinal couplings between domain-specific achievement, self-concept, and interest. Child Development, 78, 430-447.

Dweck, C. S. (2007). Is math a gift? Beliefs that put females at risk. In S. J. Ceci \& W. M. Williams (Eds.), Why aren't more women in science? Top researchers debate the evidence (pp. 47-55). Washington, DC: American Psychological Association.

Dweck, C. S., \& Bush, E. S. (1976). Sex differences in learned helplessness: (I) Differential debilitation with peer and adult evaluators. Developmental Psychology, 12, 147-156.

Eccles, J. S. (2007). Where are all the women? Gender differences in participation in physical science and engineering. In S. J. Ceci \& W. M. Williams (Eds.), Why aren't more women in science? Top researchers debate the evidence (pp. 199210). Washington, DC: American Psychological Association.

Fennema, E., \& Sherman, J. (1978). Sex-related differences in mathematics achievement and related factors: A further study. Journal for Research in Mathematics Education, 9, 189-203.

Fontayne, P., Martin-Krumm, C., Buton, F., \& Heuzé, J. P. (2003). Validation française de la version révisée de l'échelle de mesure des attributions causales (CDS II). Les Cahiers Internationaux de Psychologie Sociale, 58, 59-72.

Forsterling, F., \& Binser, M. J. (2002). Depression, school performance, and the veridicality of perceived grades and causal attributions. Personality and Social Psychology Bulletin, 28(10), 1441-1449.

Fredericks, J. A., \& Eccles, J. S. (2002). Children's competence and value beliefs from childhood through adolescence: Growth trajectories in two male-sextyped domains. Developmental Psychology, 38, 519-533.

Frome, P. M., Alfeld, C. J., Eccles, J. S., \& Barber, B. L. (2006). Why don't they want a male-dominated job? An investigation of young women who changed their occupational aspirations. Educational Research and Evaluation, 12, 359-372.

Georgiou, S. (1999). Achievement attributions of sixth grade children and their parents. Educational Psychology, 19, 399-412.

Guiso, L., Monte, F., Sapienza, P., \& Zingales, L. (2008). Culture, gender, and math. Science, 320, 1164-1165.

Hair, E., \& Graziano, W. G. (2003). Self-esteem, personality and achievement in high school: A prospective longitudinal study in Texas. Journal of Personality, 71, 971-994. 
Haugen, R., \& Lund, T. (2002). Self-concept, attributional style and depression. Educational Psychology, 22(3), 305-315.

Heller, K. A., \& Parsons, J. E. (1981). Sex differences in teachers' evaluative feedback and students' expectancies for success in mathematics. Child Development, 52, 1015-1019.

Herbert, J., \& Stipek, D. T. (2005). The emergence of gender differences in children's perceptions of their academic competence. Applied Developmental Psychology, 26, 276-295.

Heyman, G. D., \& Legare, C. H. (2004). Children's beliefs about gender differences in the academic and social domains. Sex Roles, 50, 227-239.

Hirschy, A., \& Morris, J. (2002). Individual differences in attributional style: The relational influence of self-efficacy, self-esteem, and sex role identity. Personality and Individual Differences, 32, 183-196.

Hoppe, F. (1930). Untersuchungen zur Handlungs- und Affektpsychologie. Erfolg und Misserfolg [Psychological studies of action and affect. Success and failure]. Psychologische Forschung, 14, 1-63.

Ickes, W. J., Layden, M. A. (1978). Attribution styles. In J. H. Harvey, W. J. Ickes, \& R. F. Kidd (Eds.), New directions in attribution research, Vol. 2 (pp. 119152). Hillsdale, NJ: Lawrence Erlbaum.

Inglehart, M. R., Markus, H., \& Brown, D. R. (1989). The effects of possible selves on academic achievement: A panel study. In J. P. Forgas \& J. M. Innes (Eds.), Recent advances in social psychology: An international perspective (pp. 469-477). Amsterdam: Elsevier Science Publishers.

Johnson, D. S. (1981). Naturally acquired learned helplessness: The relationship of school failure to achievement behavior, attributions, and self-concept. Journal of Educational Psychology, 73, 174-180.

Kernis, M. H., Whisenhunt, C. R., Waschull, S. B., Greenier, K. D., Berry, A. J., Herlocker, C. E., \& Anderson, C. A. (1998). Multiple facets of selfesteem and their relations to depressive symptoms. Personality and Social Psychology Bulletin, 24(6), 657-668.

Killen, M., Margie, N. G., \& Sinno, S. (2006). Morality in the context of intergroup relationships. In M. Killen \& J. Smetana (Eds.), Handbook of moral development (pp. 155-183). Mahwah, NJ: Erlbaum.

Liben, L. S., Bigler, R. S., \& Krogh, H. R. (2001). Pink and blue collar jobs: Children's judgments of job status and job aspirations in relation to sex of worker. Journal of Experimental Child Psychology, 79, 346-363.

Lightbody, P., Siann, G., Stocks, R., \& Walsh, D. (1996). Motivation and attribution at secondary school: The role of gender. Educational Studies, 22, 13-25.

Maccoby, E. E., \& Jacklin, C. N. (1974). The psychology of sex differences. Stanford, CA: Stanford University Press.

Malcom, S., Teich, A. H., Jesse, J. K., Campbell, L. A., Babco, E. L., \& Bell, N. E. (2005). Preparing women and minorities for the IT workforce: The role of non- 
traditional educational pathways. Washington, DC: American Association for the Advancement of Science.

Marini, F. (1990). Successo e insuccesso nello studio. FrancoAngeli: Milano.

Marsh, H. W. (2007). Self-concept theory, measurement and research into practice: The role of self-concept in educational psychology. Leicester, UK: British Psychological Society.

Marsh, H. W., Byrne, B. M., \& Shavelson, R. J. (1988). A multifaceted academic self-concept: Its hierarchical structure and its relation to academic achievement. Journal of Educational Psychology, 80, 366-380.

Marsh, H. W., Byrne, B. M., \& Yeung, A. S. (1999). Causal ordering of academic self-concept and achievement: Reanalysis of a pioneering study and revised recommendations. Educational Psychologist, 34, 155-167.

Marsh, H. W., Cairns, L., Relich, J., Barnes, J., \& Debus, R. L. (1984). The relationship between dimensions of self-attribution and dimensions of self-concept. Journal of Educational Psychology, 76(1), 3-32.

Marsh, H. W., \& Craven, R. G. (2006). Reciprocal effects of self-concept and performance from a multidimensional perspective. Beyond seductive pleasure and unidimensional perspectives. Perspectives on Psychological Science, 1(2), 133-163.

Marsh, H. W., \& Hattie, J. (1996). Theoretical perspectives on the structure of selfconcept. In B. A. Bracken (Ed.), Handbook of self-concept (pp. 38-90). New York: Wiley.

Marsh, H. W., \& O’Mara, A. (2008). Reciprocal effects between academic self-concept, self esteem, achievement, and attainment over seven adolescent years: Unidimensional and multidimensional perspectives of self-concept. Personality and Social Psychology Bulletin, 34, 542-552.

Marsh, H. W., \& Scalas, L. F. (2010). Self-concept and learning: Reciprocal effects model between academic self-concept and academic achievement. In B. McGaw, E. Baker, \& P. P. Peterson (Eds.), International encyclopedia of education (pp. 660-667). New York: Elsevier.

Maslow, A. H. (1954). Motivation and personality. New York: Harper and Row.

McClelland, D. C., Atkinson, J. W., Clark, R. A., \& Lowell, E. L. (1953). The achievement motive. New York: Appleton-Century-Crofts.

Meleddu, M., \& Scalas, L. F. (2009). Rendimento scolastico, fattori della personalità, processi motivazionali e sistema del sé: una rassegna sullo sviluppo degli studi. Giornale di Psicologia, 3, 297-318.

Midgley, C., Arunkumar, R., \& Urdan, T. C. (1997). Se vado male a scuola ho una scusa: le strategie di autosabotaggio. Difficoltà di Apprendimento, 3, 243-257.

Moè, A., \& De Beni, R. (2002). Stile attributivo, motivazione ad apprendere ed atteggiamento strategico. Una rassegna. Psicologia Clinica dello Sviluppo, 4 , 5-35. 
Mullis, I. V., Martin, M. O., Foy, P., \& Olson, J. F. (2008). Timss 2007: International Mathematics Report. Findings from IEA's trends in international mathematics and science study at the fourth and eighth grades. Boston: TIMSS \& PIRLS International Study Center.

National Science Foundation (2003). New formulas for America's workforce: Girls in science and engineering. Washington, DC: Author.

Nelson, J., \& Manset-Williamson, G. (2006). The impact of explicit self-regulatory reading comprehension strategy instruction on the reading-specific selfefficacy, attributions and affect of students with reading disabilities. Learning Disability Quarterly, 29, 213-230.

Newcombe, N. S. (2007). Taking science seriously: Straight thinking about sex differences. In S. J. Ceci \& W. Williams (Eds.), Why aren't more women in science? Top researchers debate the evidence (pp. 69-77). Washington, DC: American Psychological Association.

Nieuwenhuizen, A. (2001). Young Australians reading: From keen to reluctant readers (YAR). Melbourne: Australian Centre for Youth Literature.

Okolo, C. (1992). The effects of computer-based attribution retraining on the attributions, persistence, and mathematics computation of students with learning disabilities. Journal of Learning Disabilities, 25(5), 327-334.

Parsons, J. E., \& Ruble, D. N. (1977). The development of achievement-related expectancies. Child Development, 48, 1075-1079.

Powers, S., \& Wagner, M. (1984). Attributions for school achievement of middle school students. Journal of Early Adolescence, 4, 215-222.

Schmeck, R. M. (1988). Learning strategies and learning styles. New York - London: Plenum Press.

Schunk, D. H. (1991). Self-efficacy and academic motivation. Educational Psychologist, 26, 207-231.

Setencich, J. (1994). The impact of early grade retention on the academic achievement and self-esteem of seventh and eighth grade students. Paper presented at the annual meeting of the National Association of School Psychologists, Seattle, WA.

Skinner, E. A., Zimmer-Gembeck, M. J., \& Connell, J. P. (1998). Individual differences and the development of perceived control. Monographs of the Society for Research in Child Development, n.s. 254, 6(2-3), 1-220.

Smith, L., Sinclair, K. E., \& Chapman, E. S. (2002). Students' goals, self-efficacy, self-handicapping, and negative affective responses: An Australian senior school student study. Contemporary Educational Psychology, 27, 471-485.

Steele, J. (2003). Children's gender stereotypes about math: The role of stereotype stratification. Journal of Applied Social Psychology, 33, 2587-2606.

Stipek, D., \& Gralinski, H. (1991). Gender differences in children's achievementrelated beliefs and emotional responses to success and failure in mathematics. Journal of Educational Psychology, 83(3), 361-371. 
Tse, L. (2000). Student perceptions of foreign language study: A qualitative analysis of foreign language autobiographies. The Modern Language Journal, 84, 69-84.

Twist, L., Schagen, I., \& Hodgson, C. (2007). Readers and reading: The national Report for England 2006. PIRLS: Progress in International Reading Literacy Study. Slough: NFER.

Van Overwalle, F., \& De Metsenaere, M. (1990). The effect of attribution-based intervention and study strategy training on academic achievement in college freshmen. British Journal of Educational Psychology, 60, 299-311.

Weiner, B. (1985). An attributional theory of achievement motivation and emotion. Psychological Review, 92, 548-573.

Weiner, B., Frieze, I. H., Kukla, A., Reed, L., Rest, S., \& Rosembaum, R. M. (1971). Perceiving the causes of success and failure. Morristown, NJ: General Learning Press.

Weiner, B., Russel, D., \& Lerman, D. (1979). The cognition-emotion process in achievement-related contexts. Journal of Personality an Social Psychology, 37, 1211-1220.

Wolleat, P. L., Pedro, J. D., Becker, A. D., \& Fennema, E. (1980). Sex differences in high school students' causal attributions of performance in mathematics. Journal for Research in Mathematics Education, 11, 356-366.

Zimmerman, B. J. (1989). A social cognitive view of self-regulated academic learning. Journal of Educational Psychology, 81, 329-339.

\section{RiassunTO}

Si propone uno studio correlazionale su 18436 studenti di classi seconde di scuole secondarie di primo grado di nove regioni italiane, volto a esplorare alcune dimensioni motivazionali coinvolte nell'apprendimento. Si pesrseguono due specifici obiettivi di ricerca: (1) verificare la presenza di differenze in base al genere e alla regolarità negli studi, nel concetto di sé in Matematica e in Italiano e negli stili di attribuzione per la spiegazione della riuscita o del fallimento in situazioni di apprendimento; (2) testare l'associazione tra il concetto si sé in MatematicalItaliano e gli stili di attribuzione, al netto delle differenze imputabili al genere e alla regolarità negli studi. I dati sono stati rilevati attraverso il "Questionario Studente» predisposto dal Servizio Nazionale di Valutazione. Rispetto alla prima domanda di ricerca emerge che i maschi presentano un migliore concetto di sé in Matematica e riconoscono cause esterne, stabili e incontrollabili alla base dei propri successi e insuccessi, diversamente dalle fermmine che presentano un migliore concetto di sé in Italiano e valorizzano limpegno personale per la buona riuscita scolastica. Inoltre, gli studenti posticipatari presentano complessivamente un più basso concetto di sé scolastico e 
attribuzioni causali meno funzionali. Rispetto alla seconda domanda di ricerca, i risultati confermano la forte associazione tra concetto di sé e stili di attribuzione anche al netto del genere e della regolarità negli studi. Il presente studio contribuisce allo sviluppo della ricerca sulle dimensioni motivazionali dell'apprendimento e allidentificazione di possibili fattori protettivi o di rischio per il successo e l'insuccesso scolastico.

Parole chiave: Apprendimento scolastico, Attribuzioni causali, Concetto di sé scolastico, Regolarità negli studi, Studio correlazionale.

How to cite this Paper: Caputo, A. (2015). Le dimensioni motivazionali dell'apprendimento scolastico: uno studio correlazionale sul concetto di sé e gli stili di attribuzione [The motivational patterns of school learning: A correlational study on self-concept and attributions]. Journal of Educational, Cultural and Psychological Studies, 12, 143-167. doi: 10.7358/ecps-2015-012-capu 